\title{
Neural Network with Specialized Knowledge for Forecasting Intermittent Demand
}

\author{
Alexandre Crepory Abbott de OLIVEIRA ${ }^{\mathrm{a}, 1}$, Jéssica Mendes JORGE ${ }^{\mathrm{a}}$, Andrea Cristina \\ dos SANTOS ${ }^{\mathrm{b}}$ and Geraldo Pereira Rocha FILHO ${ }^{\mathrm{c}}$ \\ a Mechatronic Systems Graduate Program, University of Brasilia \\ ${ }^{\mathrm{b}}$ Department of Industrial Engineering, University of Brasilia \\ ${ }^{\mathrm{c}}$ Department of Computer Science, University of Brasilia
}

\begin{abstract}
Demand forecasting is an essential part of an efficient inventory control system. However, when the demand has an intermittent or lumpy behavior, forecasting it becomes a challenging task. Several methods have been developed to solve this issue, but nonetheless, they only consider the information about the occurrence of demand, failing to assess the drivers of the data behavior. With the current digitalization of the industry, more data is available and, therefore, the chances of finding a causal relationship between the available data and the demand increases. Considering that, this paper proposes a single-hidden layer neural network for forecasting irregularly spaced time series with attributes conveying information about the past demand, seasonality of the data and specialized knowledge about the process. The neural network proposed is compared with benchmark neural networks and traditional forecasting methods for intermittent demand using three different performance measures on actual demand data from an industry operating in the aircraft maintenance sector. Statistical analysis is conducted on comparison results to identify significant differences in the forecasting methods according to each performance measure.
\end{abstract}

Keywords. Demand forecast, intermittent demand, neural network, specialized knowledge

\section{Introduction}

There are a variety of challenges for every area in the implementation of the smart manufacturing, or smart production factory, and overcoming them requires the collaboration of different disciplines, both technical and social disciplines. The $4^{\text {th }}$ industrial revolution focuses not only on making machines intelligent with sensors and IoT (Internet of Things) but also on automating and improving decision-making processes [1]. One of these processes is inventory control, it can have its efficiency improved thought the use of Industry 4.0 technologies [2].

Inventory control is essential for the company's competitiveness in any market [3]. One of its main components is demand forecasting. Due to its importance, several forecast methods have been developed over the years. These methods yield their best results depending on the demand behavior. The demand behavior can be divided into

\footnotetext{
${ }^{1}$ Corresponding Author, Email: alex.crepory@gmail.com.
} 
four types, as follows: (i) erratic, a pattern that has a high demand size variability and does not have many zero-demands; (ii) smooth, it has a low demand size variability and few zero-demands; (iii) intermittent, a pattern that has many zero-demands and a low demand size variability; and (vi) lumpy, it has many zero-demands and a high demand size variability [4].

Different methods centered on lumpy and intermittent demand have been proposed in the literature. The single exponential smoothing (SES) [5] was commonly used to predict the demand for these types of behavior, however, it is not as accurate as newer methods [6]. One of the earlier works focusing exclusively on this topic was [6], the Croston's method (CR) was the first to separate the demand size from the demand occurrence. Later it was shown by [7] that this method was biased. [8] proposed a revision to the $\mathrm{CR}$ method to approximately correct this bias, the method is known as Syntetos-Boylan Approximation (SBA). However, these methods do not account for the item's obsolescence. To solve this problem, [9] proposed a method that is updated every period, known as TBS (Teunter-Syntetos-Babai) method.

The Machine Learning (ML) techniques are one of the promising alternatives to predict the demand behaviors [10]-[13]. These technique can identify nonlinear functions from the data sample without any assumptions about its probabilistic distribution, making them good candidates for forecasting methods [11]. [10] proposed radial and elliptical basis functions networks to forecast the demand with inputs. [11][13] adopted multilayer perceptron networks (MLP) as the forecasting method. However, these methods only considered the pattern of the demand and not the cause of the behavior.

In this paper we go further and model a multi-layer perceptron neural network with internal knowledge of the process that drives the demand. For this, the artificial neural network (ANN) was implemented on data from the indirect material replenishment process of a helicopter engine maintenance company. The dataset is composed of 19 items with different characteristics. To compare the results of the proposed NN, the following traditional methods were employed: moving average, SES, CR, SBA, and TSB. In addition, the networks from [11]-[13] were implemented. The results were compared using three different performance measures and to ensure a robust validation of the results, a statistical analysis was performed.

This work is organized as follows: Section 1 introduces a literature review of the aforementioned forecasting methods and; in Section 2, it is presented the methodology followed in this paper; Section 3 contains the forecast results and the statistical analyses performed on the results; Section 4 presents the conclusions and future works.

\section{Demand forecasting methods}

Forecasting the demand is an essential element in any inventory control. Furthermore, it is a difficult challenge when the demand in intermittent or lumpy [13]. The single exponential smoothing method is commonly used in the industry and it is considered the standard method for intermittent demand. However, it has a bias associated with it, because it sets the last demand data with the highest weight. Therefore, the forecast tends to be higher after a demand occurrence, and lower before one [6].

To minimize this bias, [6] proposed a forecasting method composed of two SES, one for the demand size and one for the interval between non-zero demands. The CR method only updates the estimations when there is a demand occurrence. Nevertheless, 
this method was also proved to be biased [7]. To overcome this bias, [8] proposed a correction to $\mathrm{CR}$ method, making it an approximately unbiased demand estimator. This method is known as Syntetos-Boylan Approximation. [8] showed the superiority of the SBA method over the CR method in an experiment of 3000 stock-keeping units in the automotive industry.

As the CR and SBA methods are only updated when there is a non-zero demand occurrence, the predictions can become inaccurate after several periods with zero demand. This happens when an item is at risk of obsolescence. This results in a positive bias in the prediction. To solve this bias, [9] developed a forecasting method, TBS, that uses the demand probability and not the demand interval, therefore, it is updated every period. For the demand size, it utilizes the same formula as the CR and SBA methods.

Apart from the traditional methods, there are forecasting methods based on machine learning techniques, specifically, artificial neural networks. ANNs are regarded as been able to provide good approximations to most functional relationships. And by adding more attributes to the model, the prediction results can be improved [11]. ANN can also model non-linear relations in the data, commonly present in the intermittent demand behavior. This non-linearity can be overlooked by most traditional methods. In addition, for the implementation of the ANNs, there is no need for any statistical distribution assumptions [10]. Altogether, the ANNs have great potential for forecasting the intermittent behavior of the demand [11].

To the best of our knowledge, the first work focusing on predicting intermittent or lumpy demand using ANNs was [10]. They utilized three different basis functions networks for predicting the demand. The networks were Gaussian radial basis function (RBF), normalized Gaussian radial basis function (NRBF), and Gaussian elliptical basis function (EBF). The number of inputs and basis functions in the hidden unit layer was optimized for each time series. They implemented two different types of prediction. The first one was forecasting the future total demand for a period; and the second type, they forecasted the demand size and the time of the next demand occurrence. Their results showed that RBF was better suited for predicting the intermittent demand compared to the other ANNs, and it had a better performance than the CR method.

[11] proposed a MLP network trained by back-propagation to predict the future demand size for the next period. The ANN had three layers, one input layer, one hidden layer, and one output layer. The input was composed of the past demand and the number of periods between the last two non-zero demands. The hidden layer was composed of three neurons, enough to approximate most complex functions [12]. They compared their ANN to the traditional methods SES, CR, and SBA. Their results indicated that their ANN was, generally, superior to the other methods. One downside of neural network is the fact that it utilizes one dataset for training and one for testing, so it is not continuously updated with new information, while the traditional methods are updated each period.

[12] adopted the same network configuration as [11], but it changed the input layer. They used as input the number of consecutive zero-demand periods instead of the number of periods between the last two non-zero demands. For comparison, traditional methods (SES, CR, SBA, and weighted moving averages) were also implemented. Their ANN had the best overall performance in the dataset compared to the traditional methods.

However, [11], [12] did not compare their networks with other networks. [13] filled this void by comparing their proposed ANN, the two networks mentioned and the traditional methods CR and SBA. Their ANN combined the inputs from [11], [12] having three inputs. In their work, they tested different configurations of networks, including the network architecture, the learning approach, and the learning method. They tested 
three types of network architecture: feedforward neural networks, time-delay neural networks, and recurrent neural networks. For the learning approach, they tested two types: back-propagation and extreme learning machines. They assessed two different types of learning modes: batch and online. We recommend seeing [13] for further information on the configurations. Their results showed that the ANNs had a significantly better performance compared to the traditional methods. Also, the ANNs trained with the back-propagation had better performance than the ones trained by the extreme learning. And considering the training with back-propagation, the ANN from [12] achieved better results in some metrics.

\section{Methodology}

The objective of this paper is to model a multi-layer perceptron neural network to predict the demand behaviors based on the internal knowledge of the process that drives the demand.

To evaluate the performance of the proposed MLP, it was compared to traditional methods and known ANNs. The selected traditional methods were: (i) Moving average; (ii) Single exponential smoothing; (iii) Croston's method; (iv) Syntetos-Boylan Approximation; (v) Teunter-Syntetos-Babai method. The ANNs selected are from [11][13]. Table 1 presents all the methods compared in this work, their compact notation, and their parameters.

Table 1. Forecasting methods, their notation, and parameters.

\begin{tabular}{|c|c|c|}
\hline Method & Notation & Parameters \\
\hline Moving average & MAX_Y & $\begin{array}{c}\mathrm{X} \text { indicates the number of weeks considered for the } \\
\text { average and } \mathrm{Y} \text { indicates the updating frequency in } \\
\text { weeks }\end{array}$ \\
\hline Single exponential smoothing & SES & One smoothing constant \\
\hline Croston's method & $\mathrm{CR}$ & Two smoothing constants \\
\hline Syntetos-Boylan approximation & SBA & Two smoothing constants \\
\hline Teunter-Syntetos-Babai method & TSB & Two smoothing constants \\
\hline ANN from $[11]$ & GUTI & $\begin{array}{l}\text { Number of hidden layers, number of hidden } \\
\text { neurons, learning rate, momentum factor }\end{array}$ \\
\hline ANN from [12] & MUKH & $\begin{array}{l}\text { Number of hidden layers, number of hidden } \\
\text { neurons, learning rate, momentum factor }\end{array}$ \\
\hline ANN from [13] & LOLL & $\begin{array}{l}\text { Number of hidden layers, number of hidden } \\
\text { neurons, learning rate, momentum factor }\end{array}$ \\
\hline Proposed ANN & CREP & $\begin{array}{l}\text { Number of hidden layers, number of hidden } \\
\text { neurons, learning rate, momentum factor }\end{array}$ \\
\hline
\end{tabular}

These methods are implemented in a dataset of 19 weekly time series company with 345 datapoints each from a helicopter engine MRO.

The methods' performances are analyzed based on three accuracy measures: MAPE (Mean Absolute Percentage Error), MPE (Mean Percentage Error) and PB (Percentage Best). The combined analysis of the three measures gives an overall perspective of the method's behavior.

To enable the comparison of the methods, statistical analyses are performed on the results of the three accuracy measures, MAPE, MPE, and PB. The first one is the rANOVA to identify if there is one method significantly different from the others. The rANOVA was chosen over the ANOVA since all the methods are evaluated on the same sample. If the rANOVA indicates that there is a difference in the methods, a pairwise 
paired t-test is conducted. This test can identify if two methods as significantly different from each other. The statistical significance level utilized for both analyses is 0.05 .

The following subsections discuss in more detail the data utilized in the project, the ANN proposed the setting of each methods' parameters and the accuracy measures.

\subsection{Data}

The modeled ANN employs an actual demand dataset from a helicopter engine maintenance company based on Rio de Janeiro, Brazil. The dataset is composed of 19 weekly demand time series with 345 observations each. To understand the demand behavior of the dataset, the squared coefficient of variation of demand $\left(\mathrm{CV}^{2}\right)$ and the average inter-demand interval (ADI) was computed. These two variables indicate the demand size erraticness and the intermittency of the demand occurrence, respectively. Based on [4], these two variables are used to categorize the behavior as erratic, smooth, intermittent, or lumpy. The threshold values for the categorization $\left(\mathrm{CV}^{2}=0.49\right.$ and ADI $=1.32$ ) were derived from a theoretical comparison of the performance of the SES, CR and SBA methods over constant lead time. Figure 1 plots the scattering of the 19 time series $\mathrm{CV}^{2}$ and $\mathrm{ADI}$ from the dataset, it also shows the threshold values for the different demand behaviors. As seen in Figure 1, most items have an intermittent behavior ( 9 items) or lumpy behavior ( 8 items). Smooth and erratic behavior correspond to 1 item each in the dataset. Therefore, the methods focusing on the intermittency of the demand are suitable for the provided data.

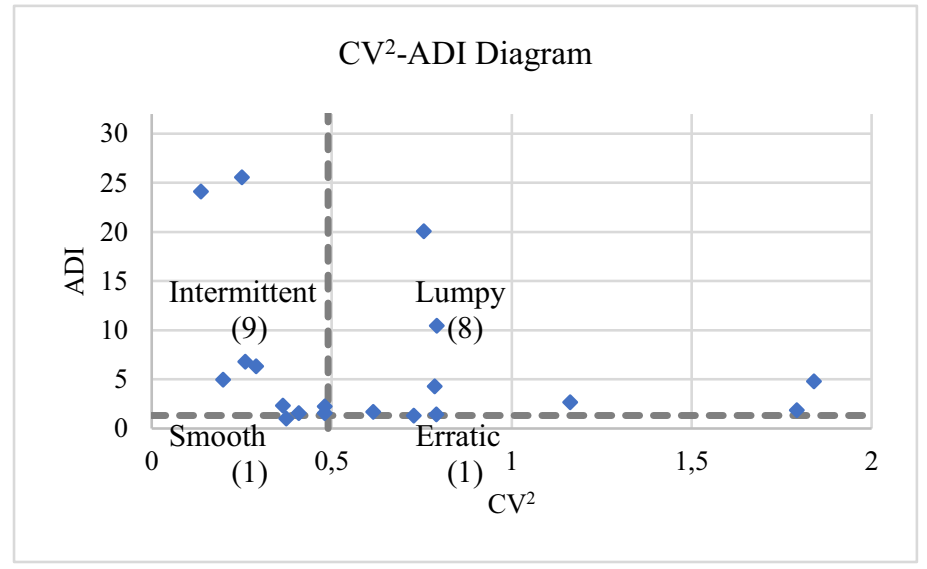

Figure 1. $\mathrm{CV}^{2}$-ADI categorization diagram.

\subsection{Proposed network}

In this paper, a neural network with specialized knowledge is implemented. It is a multilayered perceptron trained by a back-propagation algorithm. Following the guidelines proposed by [14] for multi-layered perceptron, the network is composed of three layers: (i) one input layer with 50 neurons; (ii) one hidden unit layer with three neurons; and (iii) on output unit layer.

Specialized knowledge is utilized in the input layer. This knowledge is composed of information that might impact the behavior of the demand specific to the company 
analyzed. The variables considered are: (i) year; (ii) trimester; (iii) month; (iv) engine input; (v) engine output; (vi) number of zero-demand occurrence in sequence; (vii) the number of periods between the last two non-zero demands; and (viii) the last demand. All these variables are input neurons for the first layer. In addition, the historical information of the last 4 datapoints of the variables (iv) to (viii) is also used as input. This conveys the sense of production history to the ANN. The variables (vi) and (vii) were presented by [12] and [11], respectively.

The identification of the variables was achieved by expert interviews from different areas related to production. These areas include production planning and control, inventory managing, and manufacturing.

The settings for the ANN were one hidden layer with three hidden neurons, a learning rate of 0.01 , a momentum of 0.01 , and 30,000 epochs. It was trained by a backpropagation algorithm.

The output unit layer represents the demand value of the next period and it is connected to all hidden neurons.

\subsection{Parameter settings}

The traditional methods SES, CR, SBA, and TBS have smoothing constants that need to be defined. Based on the recommendations from [6], [15] to choose the parameters in the range of 0.05 to 0.20 , we opted to use 0.1 for all the constants. For the moving average, it was decided to use the configuration MA52_26, the mean of the former 52 weeks updated every 26 weeks. The ANNs followed the same configuration as the proposed network.

Each of 19 time series was partitioned into two datasets, one for training $(65 \%)$ and one for validation (35\%), as used by [12]. The division was based on the chronology of the samples, the older datapoints were used for training and the newer ones for validation. This way, the traditional methods can be calibrated before they are utilized, and the ANNs can be trained and their parameters settled using the same dataset. The forecast results from the validation dataset were used to assess the methods' performance. One important distinction between traditional and ANNs methods is that the first is continuously updated and the second is not.

\subsection{Accuracy measures}

Different accuracy measures provide different insights about the error of the predictions. Therefore, to use only one measure would not give a complete understanding of the prediction methods [16]. We opted to use only non-scale dependent measures, so the statistical analyses on these measures would be possible [17].

The first accuracy measure utilized is the MAPE, it is the most adopted non-scale measure [12]. This measure provides insides of the bias magnitude. The standard definition of the measure uses the ratio $\left|E_{t}\right| y_{t}$, where $E_{t}$ and $y_{t}$ are the error and the actual demand in period $t$, respectively. Since there are zero demand occurrences, the following modified equation is used:

$$
M A P E=\frac{\sum_{t=1}^{n}\left|\hat{y}_{t}-y_{t}\right|}{\sum_{t=1}^{n} y_{t}}
$$


The second accuracy measure is the MPE, the ratio of the mean error and the average demand. It indicates the overall bias behavior of the error, in other words, it indicates if the method underestimates or overestimates the prediction. The equation of MPE is as follows:

$$
M P E=\frac{\sum_{t=1}^{n}\left(\hat{y}_{t}-y_{t}\right)}{\sum_{t=1}^{n} y_{t}}
$$

The last measure is the Percentage Best which is the percentage of times one method outperforms the rest considering one specific criterion, in this case, we utilized the absolute error.

\section{Results and discussions}

The means and variances of the accuracy measures for each method are presented in Table 2. For a more comprehensive analysis, this section is divided by each accuracy measure.

Table 2. Accuracy measure mean and variance for the forecasting methods.

\begin{tabular}{cccccccccc}
\hline Measure & MA52_26 & SES & CR & SBA & TBS & MUKH & GUTI & LOLL & CREP \\
\hline $\begin{array}{c}\text { MAPE } \\
\text { Mean }\end{array}$ & 1.281 & 1.277 & 1.291 & 1.268 & 1.272 & 1.151 & 1.151 & 1.152 & 1.177 \\
MAPE & 0.363 & 0.254 & 0.445 & 0.410 & 0.268 & 0.195 & 0.193 & 0.197 & 0.258 \\
Variance & & & & & & & & & \\
$\quad$ MPE & -0.044 & -0.006 & 0.015 & -0.035 & -0.004 & -0.195 & -0.196 & -0.195 & -0.161 \\
$\quad \begin{array}{c}\text { Mean } \\
\text { MPE }\end{array}$ & 0.111 & 0.017 & 0.158 & 0.142 & 0.024 & 0.077 & 0.074 & 0.078 & 0.094 \\
$\begin{array}{c}\text { Variance } \\
\text { PB }\end{array}$ & 0.217 & 0.130 & 0.025 & 0.072 & 0.076 & 0.064 & 0.138 & 0.129 & 0.149 \\
$\begin{array}{c}\text { Mean } \\
\text { PB }\end{array}$ & 0.030 & 0.012 & 0.001 & 0.008 & 0.002 & 0.009 & 0.027 & 0.007 & 0.023 \\
Variance & & & & & & & & & \\
\hline
\end{tabular}

\subsection{MAPE}

From the information of Table 2, it can be ANNs have, generally, a lower MAPE mean than the traditional methods, meaning that their absolute bias is lower. And, the MAPE variance of the ANNs tends to be lower than the other methods.

The rANOVA (Table 3) indicates that at least one of the methods is significantly different from the other $(\mathrm{p}$-value $=0.002<0.05)$. The rANOVA also indicates that the time series is also significant for the MAPE result.

Table 3. rANOVA for MAPE.

\begin{tabular}{ccccccc}
\hline Source & SS & df & MS & F & p-value & F crit (0.05) \\
\hline Time Serie & 43.010 & 18 & 2.389 & 98.211 & $2.149 \mathrm{E}-71$ & 1.676 \\
Method & 0.624 & 8 & 0.078 & 3.204 & 0.002 & 2.003 \\
Error & 3.504 & 144 & 0.024 & & & \\
Total & 47.140 & 170 & & & & \\
\hline
\end{tabular}

To identify the different methods, a pairwise paired t-test is conducted, and its result is presented in Table 4. It can be seen that the SES is statistically inferior to all the ANNs implemented, but it is not significantly different from the other traditional methods. The known conclusion that SBA is less biased than the CR method is validated once more. 
Also, the ANN proposed in this paper is the only network statistically less absolutebiased than the CR method. In contrast, the proposed ANN is the only network not different from the TBS method. There is not a significant difference between the ANNs considering the MAPE.

Table 4. Significantly different paired methods for the measures of accuracy MAPE, MPE, and PB.

\begin{tabular}{rlrlrl}
\hline \multicolumn{2}{c}{ MAPE } & \multicolumn{2}{c}{ MPE } & \multicolumn{2}{c}{ PB } \\
\hline SES & MUKH & SES & MUKH & MA52_26 & CR \\
SES & GUTI & SES & GUTI & MA52_26 & SBA \\
SES & LOLL & SES & LOLL & MA52_26 & TBS \\
SES & CREP & SES & CREP & MA52_26 & MUKH \\
CR & SBA & CR & SBA & SES & CR \\
CR & CREP & CR & MUKH & CR & SBA \\
TBS & MUKH & CR & GUTI & CR & TBS \\
TBS & GUTI & CR & LOLL & CR & GUTI \\
TBS & LOLL & CR & CREP & CR & LOLL \\
& & SBA & CREP & CR & CREP \\
& & TBS & MUKH & TBS & LOLL \\
& & TBS & GUTI & MUKH & LOLL \\
& & TBS & LOLL & MUKH & CREP \\
& & TBS & CREP & & \\
\hline
\end{tabular}

\section{2. $M P E$}

In general, the traditional methods have a better performance than the ANNs considering the MPE accuracy measure. The traditional methods resulted in MPEs closer to zero, with an overall MPE mean of -0.0147 , and the ANNs tend to underestimate the demand size, with an overall MPE mean of -0.187 .

The results from the rANOVA imply that the forecasting method and the item significantly impact the MPE of the prediction. Table 5 shows the results from the rANOVA. Moreover, the results of the t-tests in Table 4 show the paired methods that can be considered significantly different. The traditional methods SES, CR, and TBS have a smaller bias compared to all ANNs. Also, the CREP network and the SBA method are significantly different from each other. Between the ANNs, the CREP network had the closest value to zero, meaning, it had the smallest bias, however, the t-test did not indicate a significant difference between neural networks.

Table 5. rANOVA for MPE.

\begin{tabular}{ccccccc}
\hline Source & SS & df & MS & F & p-value & F crit (0.05) \\
\hline Time Serie & 5.668 & 18 & 0.315 & 5.469 & $1.304 \mathrm{E}-09$ & 1.676 \\
Method & 1.314 & 8 & 0.164 & 2.852 & 0.006 & 2.003 \\
Error & 8.292 & 144 & 0.058 & & & \\
Total & 15.274 & 170 & & & & \\
\hline
\end{tabular}

\section{3. $P B$}

While the previous accuracy measures indicate how much each method is different from the others, the percentage best expresses how many times one method is better than the others. From Table 2, the method with highest mean PB is the moving average, followed by the CREP network. 
The rANOVA for this measure indicated that the method has a significantly impact on the forecast result, as seen in Table 6 . And, as expected, the time series have no impact on the measure, because the sum the measures across the time series is approximately 1 .

The pairwise paired t-test results (Table 4) indicate that the moving average is superior to the other traditional methods (except for SES) and to the MUKH network. The CR method have an inferior performance when compared to most other methods. The PB was the only accuracy measure to indicate a significantly difference between the ANNs, the CREP and LOLL networks have significantly better results than the MUKH network.

Table 6. rANOVA for MPE.

\begin{tabular}{ccccccc}
\hline Source & SS & df & MS & F & p-value & F crit (0.05) \\
\hline Time Serie & $1.404 \mathrm{E}-31$ & 18 & $7.800 \mathrm{E}-33$ & 5.308 & 1 & 1.676 \\
Method & 0.502 & 8 & 0.063 & 4.269 & 0.0001 & 2.003 \\
Error & 2.116 & 144 & 0.015 & & & \\
Total & 2.619 & 170 & & & & \\
\hline
\end{tabular}

\section{Conclusion and future works}

To have a truly integrated smart production factory, it needs to have a transdisciplinary development. This process involves different areas from the company and will impact the way the enterprise functions. Therefore, it should integrate various expertise and it should be composed of small steps. This paper proposed an artificial neural network with specialized knowledge for forecasting intermittent demand. To the best of our knowledge, this is the first work to implement an ANN focusing on intermittent behavior with inside information on the production.

Considering the MAPE as the accuracy measure, the CREP network has a better overall performance than the traditional methods. Even though the MPE is smaller than the other ANNs, it was not able to differentiate them by the statistical means. The MPE indicates that the traditional methods have mean error closer to zero than the ANNs, and that the ANNs have a propensity to underestimate the forecast. The CREP network was the best demand predictor multiple times, having the second highest PB. A surprising result is that the moving average had a great performance. It had the highest value for PB and was not significantly different from the best methods in the other accuracy measures. Based on these results, it can be concluded that the use of ANNs and relevant attributes identified by experts have a great potential as a forecasting method for intermittent demand.

This paper did not optimize the parameters for each method, therefore, there are possibilities to improve the forecasting results furthermore. Not only the smoothing parameters for traditional methods but different network configurations for the ANNs. This way, a comparison of these methods optimized will give an insight to their possibilities.

Another possibility to improve the forecasting is to combine different forecasting methods to create hybrid models. This way, we can unite the best characteristics of each method and minimize their disadvantages. 


\section{Acknowledgement}

This study was financed in part by the Coordenação de Aperfeiçoamento de Pessoal de Nível Superior - Brasil (CAPES) - Finance Code 001.

\section{References}

[1] S. Kumar, B. S. Purohit, V. Manjrekar, V. Singh, and B. K. Lad, Investigating the value of integrated operations planning: A case-based approach from automotive industry, Int. J. Prod. Res., vol. 56, no. 22, pp. 6971-6992, 2018, doi: 10.1080/00207543.2018.1424367.

[2] J. Chen, O. Gusikhin, W. Finkenstaedt, and Y.-N. Liu, Maintenance, Repair, and Operations Parts Inventory Management in the Era of Industry 4.0, IFAC-PapersOnLine, vol. 52, no. 13, pp. 171176, 2019, doi: 10.1016/j.ifacol.2019.11.171.

[3] E. A. Silver, D. F. Pyke, and D. J. Thomas, Inventory and Production Management in Supply Chains. CRC Press, 2017.

[4] A. A. Syntetos, J. E. Boylan, and J. D. Croston, On the categorization of demand patterns, J. Oper. Res. Soc., vol. 56, no. 5, pp. 495-503, 2005, doi: 10.1057/palgrave.jors.2601841.

[5] R. G. Brown, Smoothing, forecasting and prediction of discrete time series. Englewood Cliffs, N.J., Prentice-Hall, 1962.

[6] J. D. Croston, Forecasting and Stock Control for Intermittent Demands, Oper. Res. Q., vol. 23, no. 2, 1972 .

[7] A. A. Syntetos and J. E. Boylan, On the bias of intermittent demand estimates, Int. J. Prod. Econ., vol. 71, pp. 457-466, 2001.

[8] A. A. Syntetos and J. E. Boylan, The accuracy of intermittent demand estimates, Int. J. Forecast., vol. 21, pp. 303-314, 2005, doi: 10.1016/j.ijforecast.2004.10.001.

[9] R. H. Teunter, A. A. Syntetos, and M. Z. Babai, Intermittent demand: Linking forecasting to inventory obsolescence, Eur. J. Oper. Res., vol. 214, no. 3, pp. 606-615, 2011, doi: 10.1016/j.ejor.2011.05.018.

[10] L. Carmo and J. Rodrigues, Adaptive forecasting of irregular demand processes !, Eng. Appl. Artif. Intell., vol. 17, pp. 137-143, 2004, doi: 10.1016/j.engappai.2004.01.001.

[11] R. S. Gutierrez, A. O. Solis, and S. Mukhopadhyay, Lumpy demand forecasting using neural networks, Int. J. Prod. Econ., vol. 111, pp. 409-420, 2008, doi: 10.1016/j.ijpe.2007.01.007.

[12] S. Mukhopadhyay, A. O. Solis, and R. S. Gutierrez, The Accuracy of Non-traditional versus Traditional Methods of Forecasting Lumpy Demand, J. Forecast., vol. 735, no. August 2011, pp. 721-735, 2012.

[13] F. Lolli, R. Gamberini, A. Regattieri, E. Balugani, T. Gatos, and S. Gucci, Single-hidden layer neural networks for forecasting intermittent demand, Int. J. Prod. Econ., vol. 183, no. July 2016, pp. 116128, 2017, doi: 10.1016/j.ijpe.2016.10.021.

[14] C. Xiang, S. Q. Ding, and T. H. Lee, Geometrical Interpretation and Architecture Selection of MLP, IEEE Trans. Neural Networks, vol. 16, no. 1, pp. 84-96, Jan. 2005, doi: 10.1109/TNN.2004.836197.

[15] F. R. Johnston and J. E. Boylan, Forecasting for Items with Intermittent Demand, J. Oper. Res. Soc., vol. 47, no. 1, pp. 113-121, Jan. 1996, doi: 10.1057/jors.1996.10.

[16] S. Makridakis and M. Hibon, The M3-competition: Results, conclusions and implications, Int. J. Forecast., vol. 16, no. 4, pp. 451-476, 2000, doi: 10.1016/S0169-2070(00)00057-1.

[17] R. Carbone and J. S. Armstrong, Note. Evaluation of extrapolative forecasting methods: Results of a survey of academicians and practitioners, J. Forecast., vol. 1, no. 2, pp. 215-217, Apr. 1982, doi: 10.1002/for.3980010207. 